\title{
ANÁLISIS DEL PERFIL SOCIO ECONÓMICO Y SUS EFECTOS EN EL DESARROLLO POBLACIONAL DE ALAUSÍ-ECUADOR
}

\author{
Aguilar Belén ${ }^{1}$., Illicachi Gabriela ${ }^{2}$ y Abarca Alonso ${ }^{3}$. \\ belen_aguilar07@hotmail.com ${ }^{1}$,gabyundertake@hotmail.com²y alonacional@yahoo.es ${ }^{3}$ \\ https://orcid.org/0000-0002-4039-3975 ', https://orcid.org/0000-0002-6821-780X 2, \\ https://orcid.org/0000-0003-2432-8660 ${ }^{3}$ \\ Instituto Superior Tecnológico Manuel Galecio ${ }^{1} \mathrm{y}^{2}$. \\ Instituto Superior Tecnológico Carlos Cisneros ${ }^{3}$. \\ Riobamba - Ecuador
}

Recibido (09/10/20), Aceptado (28/11/20)

\begin{abstract}
Resumen: El Cantón Alausí (Ecuador) ha experimentado factores endógenos y exógenos que han afectado el comportamiento cíclico económico y el desarrollo local. El objetivo de la investigación fue determinar el perfil socio económico de las personas del cantón Alausí. El método mixto de investigación permitió contextualizar la situación económica del cantón. Además, se determinó los factores de desarrollo económico que intervinieron en la encuesta. La muestra de estudio fue de 248 personas económicamente activas del catón Alausí. El resultado principal son la desigualdad económica existente, la carencia de recursos productivos y la mala administración de los gobiernos de turno, son factores causantes de la pobreza. Se concluye que, el desarrollo de nuevas empresas y el emprendimiento evitará el desempleo y la pobreza. A su vez, deberán promover políticas que contribuyan al cambio de la matriz productiva.
\end{abstract}

Palabras Clave: Perfil Socioeconómico, Desarrollo sostenible, Matriz productiva.

\section{ANALYSIS OF THE SOCIO-ECONOMIC PROFILE AND ITS EFFECTS ON THE POPULATION DEVELOPMENT OF ALAUSÍ-ECUADOR}

\begin{abstract}
The Canton Alausí (Ecuador) has experienced endogenous and exogenous factors that have affected the cyclical economic behavior and local development. The objective of the research was to determine the socio-economic profile of the people of the Alausí canton. The mixed research method allowed contextualizing the economic situation of the canton. In addition, the economic development factors involved in the survey were determined. The study sample consisted of 248 economically active people from the Alausí region. The main result is the existing economic inequality, the lack of productive resources and the bad administration of the governments of the moment, are factors that cause poverty. It is concluded that the development of new companies and entrepreneurship will avoid unemployment and poverty. In turn, they should promote policies that contribute to the change of the productive matrix.
\end{abstract}

Keywords: Socioeconomic Profile, Sustainable development, Productive matrix. 


\section{I.INTRODUCCIÓN}

La economía del Ecuador se ha visto caracterizada por un modelo primario exportador y extractivita [1], que han marcado la historia de los pueblos. El intento por parte del gobierno de generar riqueza a través de proyectos que contribuyan al cambio de la matriz productiva, establece que el país se convierta en productor y exportador de productos y servicios mediante la utilización de talento humano calificado y el desarrollo del conocimiento y así alcanzar un desarrollo sostenible a largo plazo. Sin embargo, la realidad económica actual indica que se espera un decrecimiento del $9.6 \%$ para este 2020 [2]. Con lo cual, ocupa el segundo lugar de las peores economías de la región.

Del mismo modo, los sectores económicos se han visto afectados debido a la baja producción y comercialización de productos, por lo que se han visto en la necesidad de reducir su nómina operativa y administrativa, lo cual ha incrementado el desempleo en el país ya que más de un millón de personas se encuentran en esta situación desde junio de este año así lo afirma [3]. Por otra parte, las ciudades al igual, que sus cantones se han visto afectadas por las políticas económicas que atraviesa el país, así como, la crisis económica desencadenada.

No obstante, el Cantón Alausí siendo una de las poblaciones más antiguas de la región, ha experimentado factores endógenos y exógenos que han afectado el comportamiento cíclico económico y por ende el desarrollo local. Posee un componente económico netamente enfocado en la agricultura que corresponde al 53\%, ya que contribuye al crecimiento del sistema económico productivo, seguido por el comercio con $37 \%$, al igual, que el turismo con 7\% Marcado por el ferrocarril, el cual, es considerado como una de las fuentes de ingresos significativa para la zona y el 3\% enfocado a actividades como la trasformación y producción de productos [4].

De la misma manera, es necesario considerar también los datos relevantes, que indican que el $71 \%$ de la población ecuatoriana está en edad de trabajar (PET), de la (PET) el 68.1\% se encuentra económicamente activa (PEA) y de la (PEA) el 96.0\% tiene empleo (considerando a los asalariados, independientes y no remunerados), y que el $4.0 \%$ están desempleados. De la misma forma, la emigración y la inmigración resultan factores importantes debido a los limitados recursos productivos con los que cuenta el cantón.

Por su parte, el gobierno de turno adoptó medidas económicas donde se establece que se modificarán subsidios de distintos sectores y otros se analizarán para su posible remoción. Por lo que, la situación económica del Ecuador, incita a realizar una investigación de la economía actual del país e inferir esa información al sector socioeconómico de Alausí, mediante la metodología descriptiva para analizar los factores demográficos del cantón que influyen en su desarrollo económico.

\section{II.DESARROLLO}

\section{A. Economía del Ecuador}

La economía ecuatoriana en el primer trimestre de 2020 experimentó un decrecimiento de $2,4 \%$ con respecto al período de 2019, según los datos de las Cuentas Nacionales publicadas por el Banco Central del Ecuador. De esta manera, el Producto Interno Bruto (PIB) totalizó USD 17.523 millones en términos constantes y USD 25.879 millones corrientes. En términos interanuales, el suministro de agua presentó una tasa de variación de $6,3 \%$. Esto se explica por el aumento de $3,7 \%$ en la producción hídrica total, principalmente de la central Hidropaute $(9,3 \%)$.

De igual forma, la actividad petrolera y minas creció $2,4 \%$ en el primer trimestre, dando como consecuencia un aumento en la producción hidrocarburífera de 2,7\%. Por otra parte, El sector de la agricultura presentó un crecimiento interanual de $1,4 \%$, del mismo modo Sobresale el aumento de 7,3\% del cultivo de banano, café y cacao y de 2,7\% del cultivo de flores [5]. Esto indica un decrecimiento en el desarrollo económico, pero a su vez un leve incremento en el modelo productor-exportador, lo que significa que, a pesar de la reducción existente, estos sectores se mantienen activos.

Para [6] la matriz productiva es la que debe dar impulso a los sectores estratégicos para que puedan ser impulsados y redefinidos, enfocados en la diversificación productiva con valor agregado, al igual, que la mejora continua y la competitividad de forma transversal en todos los sectores económicos. Las capacidades profesionales, así como saberes ancestrales y habilidades adquiridas contribuyen para establecer marcas territoriales o denominaciones de origen con la finalidad de poder ser replicadas a nivel nacional y generar empleo mediante la especialización productiva.

\section{B. Empleo}

La tasa de desempleo a nivel nacional se redujo 9.5 puntos para el segundo semestre del 2020 de modo que un millón (USD 1.000.000) de personas se quedaron desempleadas [7]. Si se contrasta esta información es significativamente mayor que la cifra registrada en junio cuando solo trescientos sesenta y seis mil (USD 366.000) personas carecían de un empleo. De la misma manera, la tasa de empleo adecuado se vio afectada y se ubicó en un $16.7 \%$, es decir, 1,8 millones de habitantes 
prescindieron de su empleo adecuado. El gobierno debe implementar políticas que aumenten el empleo formal y se controle o a su vez se regule los empleos informales.

Por otro parte, el Índice de Desarrollo Humano (IDH) permite sintetizar componentes del desarrollo económico y social de un país. Principalmente busca la redistribución de los ingresos nacionales de modo que puedan ser utilizados para ampliar el acceso a activos productivos a sectores poblacionales que aún se encuentran excluidos [8]. Los activos que se incluyen son la tierra, el agua en el campo, así como el acceso inmediato a créditos, al igual que, capacitaciones, y el apoyo con tecnologías apropiadas.

El empleo a nivel nacional ha decrecido y se ha visto delimitado por la situación económica actual del país, del mismo modo, influye directamente en los cantones ya que varios sectores se han debilitado, específicamente el agrícola, que es un eje fundamental para el desarrollo del cantón alausí, al mismo tiempo, el sector turístico por lo que varias personas han sido despojadas de su empleo lo cual se traduce en un incremento de la pobreza.

\section{Cantón Alausí}

El cantón Alausi ubicado en la provincia de Chimborazo, es considerado uno de los asentamientos más antiguos de la región. Su economía gira entorno a la actividad del ferrocarril, la consolidación del sector agropecuario y el incremento de divisas a causa de la migración. Sin embargo, con los nuevos medios de transporte el ferrocarril pasó de ser un sistema económico de transporte que permitía el intercambio entre la sierra y la costa a ser considerado un bien con potencial turístico, de igual forma, con las reformas agrarias y la distribución de tierras sin el debido financiamiento o asistencia técnica desaceleró el desarrollo de actividades agro productivas [9].

Del mismo modo, el $71.21 \%$ de la población económicamente activa (PEA) se dedica a la actividad de agricultura, la cual es la principal base económica del cantón, así como, la cría de ganado para la obtención de leche que depende de la calidad del suelo para la plantación del pasto y que este aporte los nutrientes necesarios para la alimentación del ganado. Con relación al sector turístico, el cantón cuenta con un gran potencial ya que es conocido a nivel nacional e internacional por su trayecto del tren por la nariz del diablo.

\section{Empleo}

Por otra parte, el empleo evidencia una inequidad de condiciones laborales donde las mujeres perciben el $17.5 \%$ menos de salario frente a los hombres, al igual que, el acceso a posiciones de poder de decisión con relación a la estructura económica es limitado y sigue siendo controlado por hombres. Del mismo modo, el empleo informal analiza a los trabajadores informales en base a sus ingresos donde el $52 \%$ percibe de 10,00 a 50,00 dólares semanales a diferencia del $38 \%$ que percibe de 51 a 100 dólares por semanas, al mismo tiempo, se indica que los trabajadores carecen de una estabilidad familiar.

De la misma manera, se establece que la principal causa de la pobreza es el desempleo presente en el cantón con un $47 \%$ como consecuencia de un estancamiento laboral, lo cual genera pobreza en las familias del cantón, o incita buscar fuentes de trabajo informal, que afectan al desarrollo local. Seguido de la escasez de recursos productivos que representa el $20 \%$ debido a la poca búsqueda de inversión en materia económica - productiva, ya que es obligación de todos los niveles de gobierno proveer un ambiente optimo y los servicios básicos necesarios para la realización de actividades productivas, ligado a las malas gestiones de los gobiernos de turno, las cuales también inciden en la pobreza en un $10 \%$ causado por la falta de políticas agrarias y económicas que no permitan la pérdida de los medios de producción y que contribuyan al potencial crecimiento del cantón (Ver figura 1).

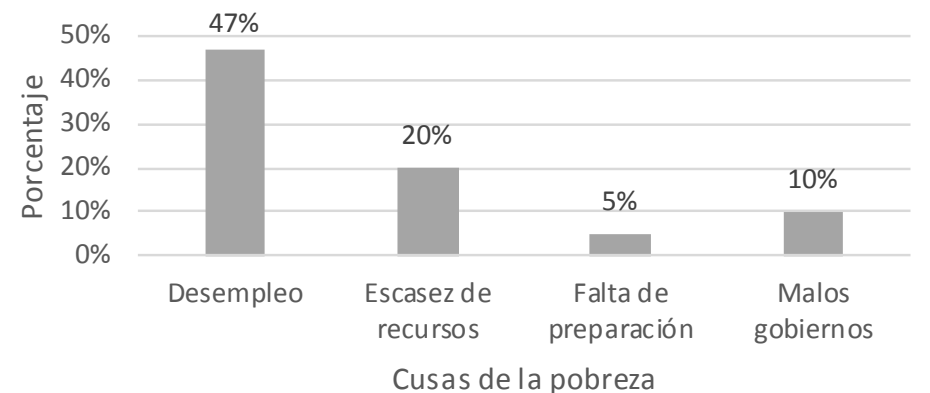

Figura 1. Causas de la pobreza en el cantón Alausi 


\section{E. Migración interna y externa}

La migración interna en el cantón Alausí se ve determinada por los limitados recursos productivos ya que esto obliga a las personas a salir del campo a la ciudad, quienes buscan nuevas fuentes de trabajo, sin embargo, en las ciudades tampoco existen las condiciones y oportunidades de conseguir un empleo estable. Por lo general este desplazamiento lo realizan cíclicamente, es decir, mientras exista oportunidades de trabajar la tierra se quedan en el canton realizando labores de siembra y cosechas y cuando estas terminan salen a las ciudades entre los meses de julio a septiembre.

Por otra parte, la migración externa del cantón se caracteriza por la falta de empleo y las condiciones de pobreza en las que se encuentran, lo cual orilla a las personas a tomar la decisión de salir del país. En Alausí $5.19 \%$ de la población optó por migrar principalmente a Estados Unidos (54\%) y España (40\%) [10]. Lo cual indica una creciente salida del país, determinada por falta de políticas económicas enfocadas en el agro, que brinden las condiciones óptimas de empleo e ingresos con la finalidad de que permanezcan en el Ecuador

\section{F. Analfabetismo}

Las condiciones de analfabetismo presentes en el cantón denotan un panorama donde alrededor del $20.69 \%$ son personas sin un nivel de educación básico o que no tuvieron la posibilidad de acceder a este derecho, la población reconocida dentro de este porcentaje son adultos mayores siendo el $36 \%$ del sexo masculino y $64 \%$ del sexo femenino. Las mujeres presentan mayores cifras de analfabetismo debido a una desigualdad de género, donde predomina el pensamiento que ella no tienen la necesidad de aprender a leer y a escribir [11].

Sin duda, la educación desempeña un rol fundamental para el desarrollo personal y colectivo del cantón Alausí ya que con el acceso a este derecho la población puede formar su criterio y a su vez desempeñar nuevas actividades de formación para emplearlas en beneficio económico, político y cultural de la población.

\section{III.METODOLOGÍA}

El estudio presentó un enfoque mixto debido a que se contextualizo a la situación económica actual, al igual que la del cantón Alausí, mediante la revisión de literatura que permitió el desarrollo de la investigación. De igual manera, se delimitaron los factores demográficos que intervienen económicamente mediante una encuesta que constó de ocho (8) preguntas. El nivel es descriptivo, puesto que, se analizó cada factor demográfico encontrado y se lo contrasta con la información obtenida a nivel nacional. En este caso se prevé un per- fil socioeconómico del cantón de Alausí provincia de Chimborazo (Ver tabla I).

\section{Tabla I. Ficha técnica de la muestra}

\begin{tabular}{ll}
\hline Parámetro & Descripción \\
\hline Muestra de estudio & $\begin{array}{l}\text { 248 personas } \\
\text { económicamente activas } \\
\text { Entorno }\end{array}$ \\
Temporalidad & $\begin{array}{l}\text { Provincia de Chimborazo } \\
\text { Encuesta }\end{array}$ \\
Método de captación & Muestreo no probabilístico \\
Procedimiento & $95 \%$ \\
Nivel de confianza & 60 \\
Cuestionarios validados & 0 \\
Excluidos & \\
\hline
\end{tabular}

El proceso de recolección de datos se lo realizó mediante la encuesta implementada, se consideró como factor delimitante el distanciamiento social establecido por la pandemia mundial (Covid-19) para la obtención de información, así como, para el desarrollo de la investigación.

\section{IV.RESULTADOS}

Los resultados obtenidos mediante la encuesta desarrollada denotan que del total de la población presente en el cantón de Alausí, el 47\% corresponde a mujeres y el $53 \%$ son hombres, lo cual indica que no existe una diferencia marcada con relación al sexo como variable demográfica, es decir cuenta con un nivel poblacional equilibrado. Sin embargo, el cantón posee diferencias de género con relación a la actividad productiva Con respecto a la variable edad, el 36\% se encuentra comprendido entre los 26 a 35 años resultando la principal población con la que cuenta el cantón Alausí, es decir, prevalece una fuerza poblacional relativamente joven que pueda impulsar el desarrollo local, de la misma manera, el 28\% está en un rango entre los 36 a 45 años al igual que, el $31 \%$ en edades de 10 a 25 años y por último, el 5\% representa a la población con edades que van de 45 en adelante, por lo cual se denota que no existe en su mayoría personas de la tercera que puedan ser consideradas como vulnerables, por otra parte la población joven puede brindar nuevas oportunidades para el desarrollo del cantón (Ver figura 2). 


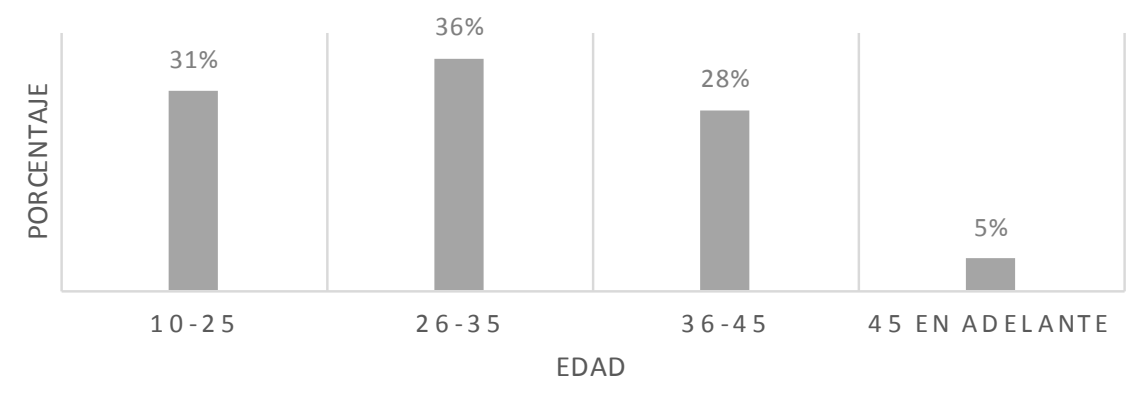

\section{Figura 2. Edad}

En el cantón de Alausí el 42\% de su población presenta un nivel académico primario y un $38 \%$ un nivel secundario. Sin embargo, el $20 \%$ de su población es analfabeta, es decir, no saben leer ni escribir, al igual que, ninguna persona cuenta con estudios superiores. Por lo cual se establece que el acceso a la educación al ser un derecho de todos los ciudadanos ecuatorianos establecidos en la constitución, debe llegar a los pueblos más necesitados con la finalidad de promover el desarrollo intelectual lo cual permita desempeñar nuevas actividades fomentando el crecimiento del cantón (Ver figura $3)$.

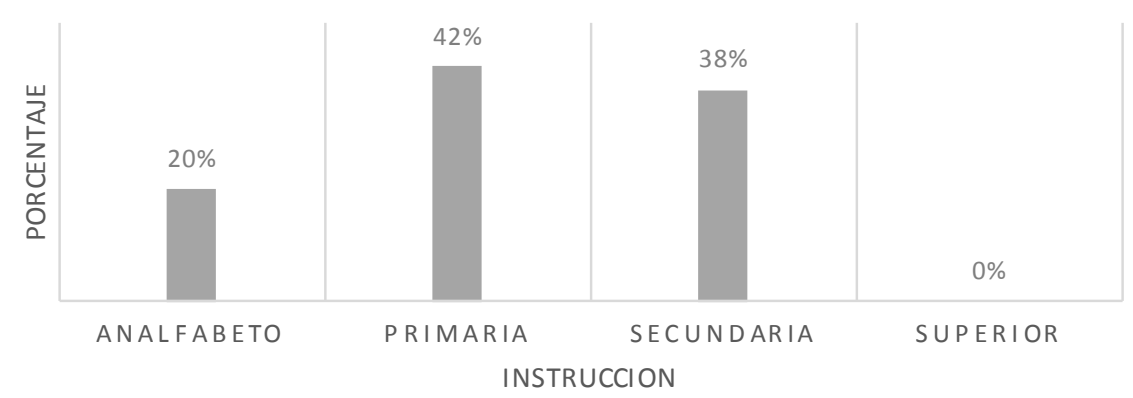

\section{Figura 3. Nivel Académico}

De igual forma, los ingresos semanales que se perciben en Alausí indican que el 36\% gana de 51 a 100 dólares por semana, el $31 \%$ únicamente gana de 10 a 50 dólares al igual que, solo el $5 \%$ percibe más de 200 dólares semanales, esto puede ser debido a la falta de políticas que regulen el ingreso salarial en base a las horas traba- jadas, o por la falta de políticas agrarias que reduzcan el costo de los recursos productivos que se necesitan para el cultivo o crianza de animales. De igual forma, por las diferencias económicas que se derivan por el desempleo y la perdida productiva (ver figura 4).

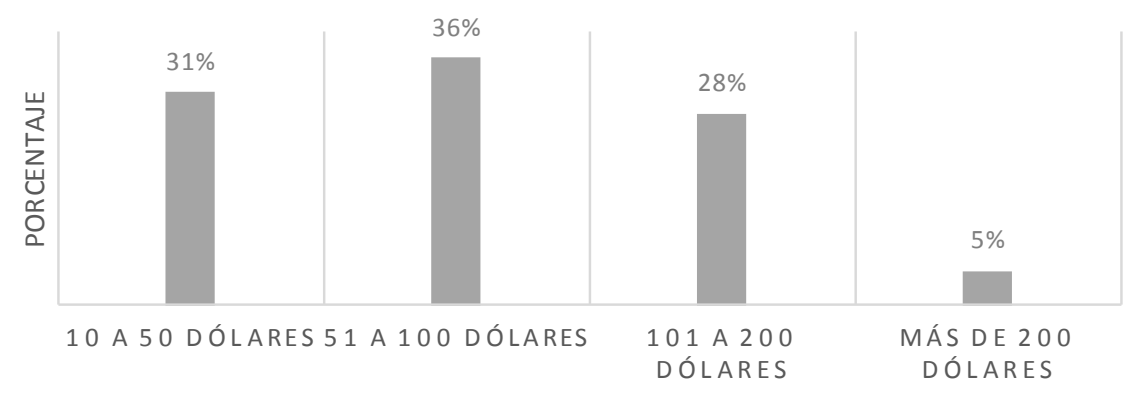

RANGO DEINGRESOS

Figura 4. Ingresos semanales 
Del mismo modo, se observa que solo el $23 \%$ cuenta con una vivienda propia, lo que establece que el $73 \%$ quienes arriendan no tienen las posibilidades para poder tener un bien inmueble, tal vez se ven limitados por el rubro de ingresos que perciben, por lo cual, no pueden acceder a créditos que financien proyectos de vivienda en el cantón. A la par el plan nacional de vivienda rural y urbana establecido en el año 2014 no generó las condiciones necesarias para que la región cuente con una residencia propia [11]. Por otra parte, el 22\% de las familias lo integran dos (2) personas, seguido del 20\% que lo integran cinco (5) personas y a su vez el $17 \%$ que lo integran tres (3) personas. El 3\% de la población la conforman familias de entre ocho o nueve personas y únicamente el $2 \%$ corresponde a un miembro, lo cual indica que no posee familia (Ver figura 5).

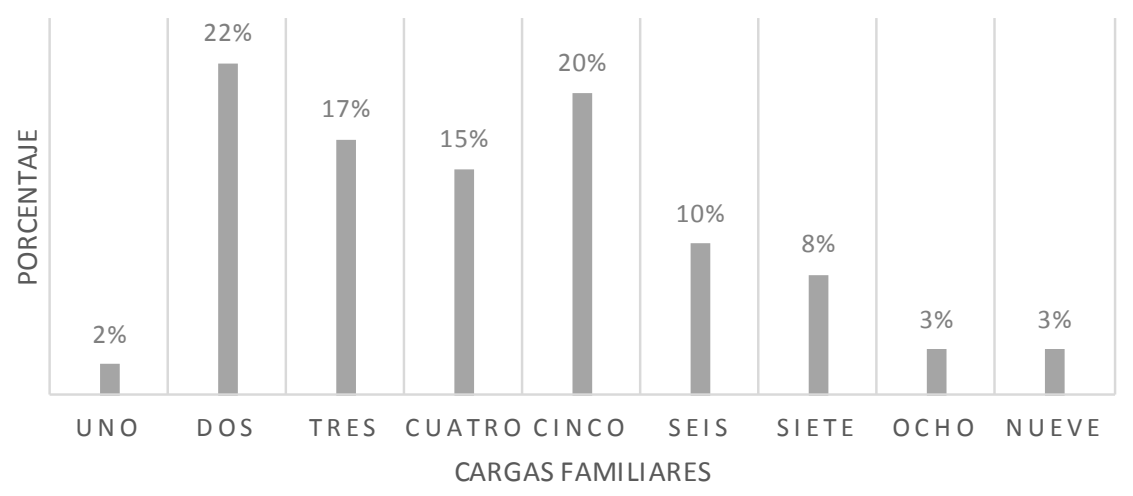

\section{Figura 5. Miembros por familia}

Se denotó que en el cantón Alausí el 95\% de la población no cuenta con un seguro de vida, esto puede estar ligado a que no forman parte del seguro social debido a que no existen muchas empresas que logren afiliar o contratar a toda la poblacion, al igual que no realizan una afiliacion voluntaria ya que no cuentan con los recursos ecnomicos para hacerlo, de la misma forma las personas que se dedican al cultivo de alimentos y actividades agrarias por lo general no cuentan con un seguro social campesino que brinde apoyo medico y economico.

Aunque en otro sentido, se determinó que el desempleo es el principal causante de la pobreza presente en el cantón Alausí (Figura 1). debido a la falta de empresas comercializadoras o industrializadas por su potencial lácteo que brinden empleo o que generen actividades laborales que apoyen a la población, del mismo modo, la escasez de recursos productivos por la falta de inversión en tecnología, al igual que, de políticas que contribuyan con la especialización y apoyo al sector agropecuario y turístico que son los principales de la región, de modo que, se ofrezca asistencia técnica. La mala gestión por parte del gobierno de turno también influye para que se incremente la pobreza, así mismo, la falta de preparación y el no poder acceder a estudios de tercer nivel para garantizar un estilo de vida digno generan desigualdad económica que está determinada por la falta de políticas económicas justas (Ver figura 6).

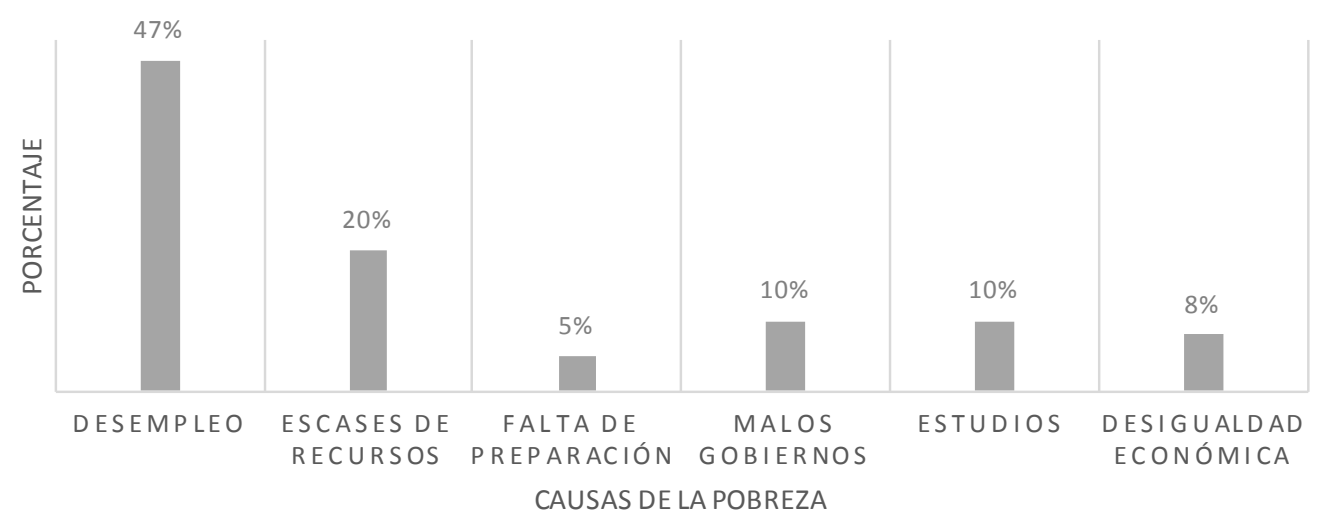

Figura 6. Otras causales de la pobreza 


\section{V.CONCLUSIONES}

La economía del Ecuador está ligada a un modelo exportador que ha ido decreciendo conforme el paso de los años debido a la baja producción y comercialización de sus productos ocasionado por la falta de políticas económicas que beneficien a los productores y que dinamicen los sectores, de manera que proporcionen recursos productivos, como tecnología o asistencia en territorio, lo cual desencadena una crisis aumentando la tasa de desempleo, la baja salarial y las causas por las cuales se origina la pobreza.

En el cantón Alusi, el sexo masculino es predominante con edades comprendidas entre los 26 a 35 años de edad, de los cuales el $20 \%$ es analfabeto y ninguno cuenta con estudios de educación superior, por lo que se debe promover el desarrollo intelectual, de modo que se fomente la consolidación de nuevas empresas que generen actividades económicas de manera, que se reduzca el desempleo al igual que promover políticas que contribuyan al cambio de la matriz productiva, en sectores como el agropecuario y así lograr a través de cadenas productivas obtener mayor número de productos derivados. Del mismo modo, el GAD parroquial debe otorgar y garantizar los medios propicios para realizar actividades productivas, para que no se ponga en riesgo la base económica del cantón Alusí y no se deriven problemas ligados principalmente a la pobreza y falta de servicios productivos

Finalmente, la desigualdad económica por los ingresos semanales percibidos, la carencia de recursos productivos para el desarrollo agricultor y la mala administración de los gobiernos de turno por la falta de políticas, son factores que ocasionan la pobreza en lo pobladores del cantón y que a su vez derivan en el aumento del desempleo ligado a una desigualdad de género que está asociado al pensamiento machista que no permite el crecimiento femenino en ámbitos, educati- vos, sociales y culturales.

\section{REFERENCIAS}

[1]R. Aguas, «Petroleum: Social and economic effect in Ecuador,» Journal og science and research, pp. 29-32, 2016.

[2]Banco Central del Ecuador, «Cuestiones Económicas,» Quito, 2017.

[3]M. Viterí y M. Tapia, «Economía ecuatoriana: de la producción agrícola al servicio,» Revista Espacios, p. 5, 2018.

[4]S. Crow, «La PEA informal y su influencia econòmica en el canton Alausi-Chimborazo,» Instituto tecnologico superior Manuel Galecio, pp. 1-3, 2018.

[5]H. Loor, D. Ureta y G. Rodriguez, «Analisis del contexto socio-economico, comercial, financiero e internacional de las pymes Ecuatorianas,» Revista cienticfica Ecociencia, vol. 5, no 4, pp. 1-21, 2018.

[6]J. Jiménez, «Movimiento de Economía Social y Solidaria de Ecuador. Circuitos Económicos Solidarios Interculturales,» Revista de la Academia, vol. 21, pp. 101-128, 2016.

[7]A. Serrano, «Análisis de condiciones de vida, el mercado laboral y los medios de producción e inversión pública,» 2013.

[8]F. Pilay, G. Cárdenas y A. Guillén, «Economía, social y solidaria, nueva forma de hacer economía,» Sathiri, vol. 12, no 1, p. 48, 2017.

[9]Gobierno parroquial de Alusí, «Plan de Ordenamiento territorial del Cantón Alausí,» 2019.

[10]Gobierno Autónomo Descentralizado de la Parroquia Bellavista, «Plan de Desarrollo y Ordenamiento Territorial Parroquial Rural de Bellavista,» Quito, 2015. [11]Ministerio de Desarrollo Urbano y Vivienda, «Programa nacional de vivienda rural y urbano marginal julio 2014,» Quito, 2014. 